\title{
Contraste de un modelo de gestión del conocimiento en una universidad pública del centro de México
}

\section{Contrast of a knowledge management model in a public university in central Mexico.}

\author{
Cruz García Lirios \\ UAEMEX \\ cgarcial213@profesor.uaemex.mx \\ Javier Carreón Guillén \\ UNAM \\ javierg@unam.mx \\ Rosa María Rincón Ornelas \\ USON \\ rosa.rincon@unidon.mx \\ Eyder Bolivar Mojica \\ USANTOMAS \\ eyder.bolivarmo@ustamed.edu.co \\ Arturo Sanchez Sánchez \\ UATX \\ artuto.sanchez.s@uatx.mx \\ Gilberto Bermudez Ruiz \\ UANAHUAC \\ gibe_gil@hotmail.com
}


A menudo, las organizaciones generadoras de conocimiento han sido estudiadas desde la influencia de su cultura, estructura y procesos flexibles e innovadores, 0 bien desde la influencia de sus liderazgos y la formación continua de sus talentos. En el presente trabajo, más bien se investigó una institución de educación superior desde los temas establecidos en la literatura especializada de 2010 a 2018, los discursos de informantes clave y tanto la confiabilidad como la validez de un instrumento que midió sus factores subyacentes. Para tal propósito, se realizaron tres investigaciones: una documental, otra cualitativa y una correlacional, a fin de poder especificar un modelo para el estudio del fenómeno en comento. Los resultados muestran una prevalencia de la generación del conocimiento como un constructo de segundo orden y una representación de sus dimensiones en torno a las categorías de "alianzas", uredes" y "climas», así como una estructura factorial en torno a la motivación, la innovación y la comunicación de oportunidades en función de recursos y capacidades. Se advierten líneas concernientes a la empatía, el compromiso y la satisfacción como factores que incrementarían el porcentaje de la varianza total explicada, que fue del $54 \%$.

Palabras clave: demandas, recursos, emprendimiento, innovación, conformidad

Often, knowledge-generating organizations have been studied from the influence of their culture, structure and flexible and innovative processes, or from the influence of their leadership and the continuous training of their talents. In the present work, a higher education institution was well researched from the topics established in the specialized literature from 2010 to 2018, the discourses of key informants and the reliability as well as the validity of an instrument that measured its underlying factors. For this purpose, three investigations were carried out: One documentary, another qualitative and another correlational in order to specify a model for the study of the phenomenon in question. The results show a prevalence of knowledge generation as a second order construct and a representation of its dimensions around the categories of "alliances», "networks" and "climates", as well as a factorial structure around the motivation, innovation and communication of opportunities based on resources and capabilities. Lines concerning empathy, commitment and satisfaction are seen as factors that would increase the percentage of the total variance explained, which was $54 \%$. 


\section{Introducción}

El objetivo del presente estudio fue contrastar un modelo para el estudio de la gestión del conocimiento mediante el establecimiento de la confiabilidad y la validez de un instrumento que midió tres dimensiones relativas a la motivación, la innovación y la comunicación, así como el contraste de la hipótesis nula relativa a la existencia de diferencias entre la estructura de las variables del fenómeno y las observaciones de las relaciones entre sus indicadores.

La gestión del conocimiento, para los fines del presente trabajo, alude a la codificación de las habilidades y de los conocimientos en torno a la producción y la transferencia de saberes e información de organizaciones hacia grupos o personas que toman decisiones para llevar a cabo la optimización de recursos, o bien, la innovación de procesos (García, 2018). De esta manera, la gestión, producción y transferencia del conocimiento desde la psicología de las organizaciones es un proceso cíclico e integral en el que liderazgos y talentos determinan estilos de innovación, motivación y comunicación orientados hacia la creación del conocimiento. Se trata de un proceso de emprendimiento constante que puede iniciarse desde la gestión, pero a menudo influido por la producción o impulsado por la transferencia del conocimiento de una organización o grupo a otro (Carreón, 2016).

Pues bien, en tanto proceso encadenado, la gestión, la producción y la transferencia del conocimiento suponen una oportunidad develada y a disponibilidad del capital humano, principalmente el intelectual ya que, si bien las organizaciones dedicadas a la creación del conocimiento generan sus propias iniciativas y propuestas, son el talento y el liderazgo humano los que identifican las oportunidades externas e internas a las organizaciones (Vázquez, Carreón \& Sánchez, 2017).

De esta manera, la psicología y la economía dotan de significado y sentido al trabajo social, que estudia a las instituciones estatales y las organizaciones civiles dedicadas a la creación del conocimiento como instrumento de desarrollo local; no obstante, ello implica que el proceso de gestión, producción y transferencia de conocimiento sea exclusivo de entidades que cuentan con el capital humano e intelectual capaz de diversificar las oportunidades y los recursos con la finalidad de adquirir una utilidad (García, 2017).

Más bien, desde la óptica del trabajo social, la creación del conocimiento es parte consustancial de las normas, valores y saberes que no solo resignifican y reorientan las propuestas de innovación, comunicación y motivación de una entidad a otra, sino que, además, suponen una instancia de satisfacción que estaría generada por la empatía y el compromiso (García, Quintero \& Carreón, 2017). Es así como la gestión, producción y transferencia de conocimiento es asumida desde el trabajo social como una dinámica de interrelación del capital humano e intelectual con el capital social. Ello quiere decir, desde una visión psicológica, que se trata de un clima integral colaborativo e innovador; mientras que, desde la economía, supone la inclusión de una externalidad al capital humano e intelectual que podría ser el capital solidario (Pérez, Valdés \& García, 2017). 


\section{Teoría de la creación del conocimiento}

Las organizaciones se diferencian por la formación de su capital humano y su enfoque en tres dimensiones de competencias: 1) lo que la organización desea o quiere hacer alude a principios normativos en los que la cultura organizacional predispone a los directivos y empleados a considerar las tradicionales formas de producción, que son vistas como modelos a seguir, siempre y cuando se asemejen a los estilos de liderazgo y comportamiento organizacional dominantes en las empresas; 2) lo que la organización sabe hacer y que supone un conjunto de habilidades y conocimientos sustentados en valores y creencias que, al incidir en decisiones de producción, calidad o competitividad, restan importancia a la generación de conocimiento o la gestión de tecnologías y procesos; y 3) lo que la organización es capaz de hacer, que está delimitado por la producción de conocimiento más que por su gestión, la formación de su capital humano más que por la satisfacción de sus talentos y el establecimiento de metas antes que por cumplir con objetivos, así como por la posibilidad de cambios antes de que los propósitos y las estrategias se agoten (Sánchez, Quintero, Sánchez, Fierro \& García, 2017).

La teoría de las alianzas estratégicas advierte que las demandas del mercado al sobrepasar los recursos de la organización las inducen a establecer alianzas estratégicas de cooperación a fin de poder incrementar las capacidades de las mipymes con el apoyo de trasnacionales, o bien desarrollar estrategias competitivas a través de la transferencia de tecnología y la implementación de la calidad de los procesos y los productos. De este modo, las mipymes sobreviven al proceso de selección y se suman al efecto multiplicador de inversiones, transferencias y procedimientos (Rodríguez, Moreno, De Rivas, Álvarez \& Sanz-Vergel, 2010).

Por su parte, la teoría de las redes de conocimiento señala que a partir del advenimiento de las tecnologías de información y comunicación (TIC), la competitividad y el establecimiento de un clima de relaciones, emerge la sistematización de recursos internos como externos con la finalidad de establecer un cambio organizacional orientado al desarrollo de procesos y productos, así como a la implementación de criterios y protocolos de calidad. En el caso de la adopción de tecnología, supone una transferencia de habilidades y conocimientos que se pueden establecer entre los institutos y las mipymes con el concurso del Gobierno local o federal, principal promotor y auspiciador de ferias de inversión o fomento emprendedor, así como de formación, prácticas y empleo (Torrelles et al., 2011).

En tal sentido, la teoría del clima de innovación supone que, en el marco de las $\mathrm{TIC}$, las organizaciones establecieron alianzas estratégicas y redes de conocimiento con la finalidad de potencializar su capital humano, intelectual e innovador. La prevalencia del clima de relaciones y el clima de innovación con respecto al clima de tareas y el clima de apoyos propició la creación de conocimientos y valores agregados a los productos y los procesos organizacionales, incrementando su calidad, diversidad y complejidad. Así, la difusión de innovaciones no solo determina el consumo, sino además el establecimiento de sectores tales como: innovadores, seguidores, precoces, tardíos y rezagados (García, Hernández \& Hernández, 2017).

Cabe señalar que la gestión del conocimiento es establecida por el estilo de 
liderazgo. De esta manera, cuatro dimensiones relativas al aprendizaje, el liderazgo, la cultura y el conocimiento generan cuatro factores: tácito a tácito, explícito a tácito, explícito a explícito y de tácito a explícito (García, Bustos, Carreón \& Hernández, 2018).

Asimismo, la creación del conocimiento, en su fase de gestión, supone la emergencia de liderazgos que, al diseminar sus estilos de comunicación y motivación, amplifican la red de conocimiento, pero limitan la competencia (Villegas, García \& Hernández, 2018). En un segundo escenario de la creación del conocimiento, la gestión se establece a partir de la competencia entre los liderazgos tradicionales y los liderazgos emergentes. Es decir, los estilos adhocráticos-burocráticos coexisten con estilos de flexibilidad postburocrática. Luego, en un tercer momento, la gestión del conocimiento se establece a partir de la reducción de un liderazgo tradicional o emergente en relación con la consolidación del liderazgo triunfador y otro liderazgo emergente. Se trata de un ciclo de cooperación, alianzas estratégicas y comunicación bilateral que garanticen una motivación permanente de las expectativas de los talentos (Carreón, Hernández, García \& Bustos, 2017). En la última fase, la gestión del conocimiento se establece por tríadas que comandan la toma de decisiones en función de acuerdos y corresponsabilidades. Se trata de una nueva cultura organizacional: flexible e innovadora por su grado de empatía y compromiso, centrada en una estructura horizontal, la comunicación bilateral y la motivación bidireccional.

Es así como la gestión y la innovación en organizaciones dedicadas a la producción y la transferencia de conocimiento se estructuran en cuatro ejes y trayectorias:

- De la innovación a su difusión. El emprendimiento es una instancia que está asociada a la agenda organizacional. En ese sentido, la innovación es un tema central en tanto genera opiniones a favor y en contra de su implementación porque anuncia el cambio organizacional y, con ello, el surgimiento de la resistencia al cambio. Por consiguiente, el emprendimiento de una innovación es un reto que los actores asumen como amenaza o como oportunidad en el marco del cambio organizacional.

- Del emprendimiento a la adopción a través de la difusión. Si el balance organizacional permite la aceptación e implementación de una tecnología, entonces los grupos a favor de la utilidad toman decisiones con un alto riesgo, las cuales estarían influidas por la motivación de líderes transformadores más que autoritarios; empero, el emprendimiento de una tecnología o proceso puede implementarse a través de la imposición más que de la negociación o la consulta (Carreón, Hernández, Quintero, García \& Mejía, 2016).

- De la innovación a la implementación. Si el clima organizacional de tareas prevalece sobre el clima organizacional de relaciones, entonces se trata de un caso de emprendimiento e implementación forzados en el que talentos y líderes carecen de voz y voto, siguiendo las directrices preestablecidas, de forma que el proceso está determinado sin considerar la difusión para llegar a la adopción. Se trata de un modelo en el que los riesgos son asumidos por quien toma la decisión de implementar la innovación tecnológica o la innovación procesual.

- De la innovación a la evaluación a través de la difusión y la adopción, o bien, a 
través de la implementación. En la primera opción, la decisión de consensuar la adopción de una tecnología o proceso supone una corresponsabilidad en el establecimiento de una agenda para evaluar los costos y beneficios, alcances y límites del acuerdo entre talentos y líderes (Carreón \& García, 2017). En la segunda opción, la implementación supone un proceso mediador de los efectos del emprendimiento y la innovación sobre la creación del conocimiento. Un aumento en la implementación supone un incremento causal entre la confianza, el compromiso, el emprendimiento, la innovación, la productividad, la competitividad y la satisfacción (García, 2019a).

La innovación organizacional ha sido establecida a partir de la diferencia entre demandas y recursos, oportunidades y capacidades. En ese sentido, un aumento de los factores externos y una reducción de los factores internos suponen un incremento de la innovación, aun cuando esta subyace a las relaciones más que a las tareas, ya que implica una inconformidad ante el clima de relaciones y el clima de tareas establecidos.

Roger (1983) definió la innovación como la difusión de información y sus efectos inmediatos, tempranos, tardíos y rezagados por parte de los potenciales consumidores. En ese sentido, estableció como criterio esencial de una innovación a la difusión y su inclusión en la agenda de consumidores potenciales. Es decir, una innovación no solo es una alternativa de solución nueva, eficiente, eficaz y efectiva, sino que además es un instrumento de persuasión y disuasión de otras innovaciones. Por consiguiente, los adoptadores inmediatos y tempranos de la innovación asumen más riesgos de oportunidad que los consumidores tardíos o rezagados en la adopción de la innovación. Adicionalmente, la innovación es «la producción o adopción, asimilación y explotación de una novedad de valor agregado en las esferas económicas y sociales; la renovación y la ampliación de los productos, servicios y mercados; desarrollo de nuevos métodos de producción; y el establecimiento de una nueva gestión sistemas. Es a la vez un proceso y un resultado» (Crossan \& Apaydin, 2010, p. 1155).

En las organizaciones creadoras de conocimiento, la innovación es contraparte de la optimización de procesos y estaría ligada a la traducción del conocimiento, ya que se gesta en talentos especializados y debe diseminarse al resto del capital intelectual (De Mello, Marx \& Salerno, 2012). Es decir, una nueva optimización puede ser considerada una innovación, pero en cuanto a las redes colaborativas donde prevalecen la motivación bidireccional y la comunicación eficaz, las innovaciones deben ser más que una mejor optimización de recursos (Guedes, Loiola, Andrade \& Da Costa, 2015). Incluso, una innovación debe aplicarse en los procesos de tareas, apoyos y relaciones más que en los recursos, capacidades y productos (Conto, Fiore \& La Sala, 2013).

Por consiguiente, en las instituciones de educación superior (IES), las innovaciones son asumidas como códigos de conocimiento que derivarían del tipo de cultura organizacional (Cherubini, Barbieux, Reichert, Tello \& Zawislak, 2016). Así, en las culturas del éxito, la innovación es un dispositivo de gestión, producción y transferencia más que una novedad en los productos, capacidades o recursos (Cleger, 2016).

La teoría de la innovación organizacional, siguiendo los postulados de la teoría de la difusión de innovaciones, supone una instrumentación nueva que se cristaliza en valor 
agregado, pero a diferencia de la difusión de innovaciones que plantea una propensión a la utilidad y los riesgos, la innovación organizacional atiende al balance entre demandas y recursos, oportunidades y capacidades.

De este modo, la innovación organizacional es en primera instancia una difusión innovadora motivacional, instrumentada por liderazgos transformadores, pero en la cristalización del emprendimiento, la innovación organizacional genera ventajas competitivas y valores agregados que desembocan en el reconocimiento y el prestigio (García, Carreón, Hernández, Bustos \& Quintero, 2017). En consecuencia, el clima organizacional que se genera al interior de la organización propicia el surgimiento de difusiones innovadoras, pues aun cuando los liderazgos no sean trasformadores, la innovación es organizacional porque se construye a partir de las percepciones de demandas y recursos, oportunidades y capacidades (Carreón et al., 2017).

En tal proceso, la adopción de la innovación no solo implica su implementación, sino además su implementación ajustada a los requerimientos de talentos y liderazgos. En otras palabras, la innovación organizacional, a diferencia de otras innovaciones, es una construcción permanente de las expectativas, necesidades, capacidades y oportunidades de una organización que puede asemejarse a otros conglomerados, pero adquiere un sello distintivo en la generación de creencias de rechazo o aceptación de la innovación, e incluso el seguimiento de la implementación es resultado de creencias a favor o en contra de la utilidad, los costos y los riesgos de adoptar la innovación (Sánchez et al., 2017). Por lo tanto, la teoría de la innovación organizacional no solo anticipa escenarios de climas organizacionales en contra o a favor de la innovación, sino que además prevé quiénes toman las decisiones de adopción, implementación, utilización, desarrollo y evaluación.

Inclusive, la teoría de la innovación organizacional sería capaz de predecir la emergencia de iniciativas que cuestionen los beneficios de la innovación, así como el surgimiento de propuestas de sustitución de la innovación. En el caso de la difusión de la innovación, el clima de relaciones sería evaluado conforme al clima de tareas y no al revés.

\section{Estudios de la creación del conocimiento}

Los estudios en organizaciones creadoras de conocimiento advierten que prevalecen diferencias en cuanto a sexo, edad, escolaridad y estatus.

En las organizaciones, la relación entre culturas, climas, competencias y comportamientos incide en la gestión de liderazgos, conocimientos, tecnologías y habilidades que, en referencia a valores y creencias, privilegian la obtención de logros por sobre la satisfacción o la colaboración.

En el caso de las competencias, estas parecen frenar la diversidad de ideas que el alumno genera al momento de llevar a cabo una actividad. En efecto, la identidad está vinculada con el acceso a información poco estructurada, pero consistente con un aprendizaje significativo. El estudiante aprende en base a un repertorio de símbolos y significados producidos por el contexto en el que se encuentra y traducidos por el grupo al que pertenece o quiere pertenecer (Gómez, 2010).

Empero, las competencias relativas a los grupos han sido definidas a partir de una lógica individual, ya que están indicadas por oportunidades, capacidades y 
responsabilidades inherentes a la persona más que a su interdependencia con los demás integrantes de equipo. Incluso, la autonomía de las instituciones incide en la elección de la escuela y, por consiguiente, en la formación profesional a través de un currículum particular. Es decir, las competencias en su modo genérico están influidas por estructuras y sistemas organizacionales que pueden inhibir o facilitar el desarrollo de competencias más específicas. Es así como los procesos de capacitación y adiestramiento para el desarrollo de competencias relacionadas con las ventas son inhibidos por la cultura de la organización tradicional (Prieto \& Villamor, 2012).

En ámbitos tecnológicos las organizaciones desarrollan competencias relativas al empleo de ordenadores y programas que en principio los hacen más valiosos por su utilidad y más competitivos por su innovación. En esa línea, el desarrollo de competencias relacionadas con el liderazgo o la planificación es esencial para entablar negocios basados en alianzas estratégicas (Secanella, 2011).

No obstante, el clima organizacional, al verse afectado por la subcontratación de personal especializado, reduce las oportunidades y con ello las capacidades y responsabilidades de los empleados cuyos puestos suponen funciones ordinarias $y$, por ende, son susceptibles a su reemplazo. O sea, a medida que las organizaciones complejizan sus procesos, demandan mayores funciones a sus empleados $y$, al acelerar la productividad y el control de calidad, recurren a la contratación alterna, inhibiendo con ello la cultura laboral, la confianza y la lealtad de sus talentos potenciales. En ese contexto, las competencias organizacionales son asumidas como habilidades más que como conocimientos y valores. Por ello, la organización pasa de ser productora de valor a reproductora de procesos, reduciendo drásticamente sus ventajas competitivas (Molina, Cruz, Carreón \& Rojano, 2017).

En el ámbito educativo las competencias laborales son el resultado de una serie de procesos deliberados en los que la capacitación y el adiestramiento son instrumentos de gestión de conocimiento determinados por las demandas del mercado y el contexto económico en el que se desenvuelven las organizaciones. Empero, las oportunidades parecen derivar de escenarios en los que tanto la cultura como el clima laboral son asequibles con las capacidades y responsabilidades de sus directivos y empleados. En tal sentido, la formación de liderazgos supone la emergencia de estrategias orientadas a la consecución de objetivos más que a la satisfacción laboral, aunque pueden significar procesos de emprendimiento. Las organizaciones son también consideradas como extensiones de la cultura y, en este tenor, las metas están ancladas al clima de relaciones tanto como al clima de tareas (García, Carreón \& Hernández, 2017).

Si la cultura y el clima organizacional, a pesar de ser disímbolos, inciden en la formación profesional, entonces el aprendizaje de competencias está más orientado al saber hacer que al saber ser. En aquellas profesiones en que las habilidades sociales e interpersonales son fundamentales para explicar la emergencia de nuevas formas de colaboración, las competencias están centradas en el aprendizaje de tecnologías y programas vinculados con la práctica profesional más que con la producción de conocimiento. Es decir, la dimensión profesional de las competencias está enfocada en el desarrollo de técnicas de procesos que supondrían un incremento en las habilidades mientras que los conocimientos 
y valores permanecen sin cambios, y la formación profesional de capital humano estaría definida por los avances tecnológicos más que por el desarrollo humano o la responsabilidad social y ambiental de las organizaciones (García et al., 2015).

A pesar de que la optimización de procesos y minimización de residuos son consideradas ventajas competitivas, las organizaciones parecen transitar hacia la tecnificación y, en consecuencia, la formación de sus talentos incluye un currículum de habilidades más que de conocimientos o valores. En tal escenario, las organizaciones parecen generar oportunidades de capacitación y adiestramiento técnico, incidiendo en las capacidades computacionales y disminuyendo la responsabilidad que implica el consumo de tecnologías y energía (Carreón, 2016).

Las diferencias entre los estilos de formación profesional centrados en las habilidades más que en la producción de conocimiento o la responsabilidad social y ambiental también puede observarse en las relaciones entre directivos y empleados. En el marco de las instituciones educativas, las diferencias organizacionales se traducen en diferencias de aprendizaje en cuanto a competencias se refiere. De ese modo, los líderes tienden a resaltar más los logros que el aprendizaje de habilidades o el respeto a los valores de la organización.

En contraste, los empleados enfatizan más el aprendizaje de estrategias en cuanto a la eficiencia de procesos que el logro de metas o la implementación de una cultura laboral. Por su parte, los directivos que enaltecen los valores de la empresa tienden a minimizar los programas de adiestramiento y capacitación, aunque los empleados adquieran una serie de técnicas y estrategias; o, en todo caso, cumplan con los propósitos de colaboración, productividad, calidad y competitividad. La gestión de una identidad prolifera sobre la producción de conocimiento (Genesi, Romero \& Tinedo, 2011).

Cabe resaltar que las organizaciones que desean, saben y son capaces de llevar a cabo estilos de liderazgo y elaboración de procesos están más cercanas a una cultura organizacional en la que las competencias son recursos de cambio más que de conformidad o satisfacción (Henao \& Londoño, 2012, p. 204). En esa línea, las competencias organizacionales parecen vincular normas, valores y creencias con los conocimientos y habilidades que indicarían una cultura organizacional centrada en los procesos más que en la producción y gestión de conocimiento; sin embargo, las competencias resaltan el clima de tareas como su sello distintivo, ya que la implementación de tecnologías implica procesos y programas de adiestramiento y capacitación. Así, las habilidades antes que los valores describirían a las organizaciones tecnificadas acordes a la demanda del mercado en detrimento de su planificación, sus estrategias y alianzas, las cuales solo serían intermediarias del propósito esencial de insertar a la organización al concierto global.

No obstante, en el ámbito local, la responsabilidad social y ambiental está inmersa en los requerimientos que las trasnacionales solicitan a sus maquiladoras y distribuidoras, mientras que la tecnificación de procesos solo es un valor agregado a los sistemas de gestión. Es patente, pues, que la cultura empresarial de las pymes devela su querer hacer más que su saber hacer o su capacidad de hacer (Sánchez et al., 2017a).

Los estudios de la innovación del conocimiento incluyen procesos organizacionales en torno a la gobernanza de las innovaciones. Se trata de un proceso en el que el seguimiento y la evaluación constante por parte de talentos y líderes suponen un proceso 
de emprendimiento no equiparable a la adopción, sino más bien al establecimiento de una agenda. Esto es así porque la gobernanza supone un diálogo constante entre los actores, así como una corresponsabilidad entre las partes (García et al., 2015). Sin embargo, cuando la decisión de implementar la tecnología o el proceso es más bien asumida unilateralmente, entonces la autoevaluación prevalece sobre cualquier propuesta de mejora al sistema tecnológico o procesual adoptado.

De este modo, el estudio de la innovación organizacional puede llevarse a cabo a partir del contraste de las hipótesis relativas a las trayectorias de relaciones de dependencia entre el emprendimiento, la difusión, la adopción, la implementación y la evaluación de un sistema tecnológico o proceso organizacional que repercuta en la generación de ventajas competitivas y valores agregados (Molina et al., 2017).

Por otro lado, las culturas organizacionales, en referencia a las competencias laborales, están vinculadas por climas de colaboración y tareas que reflejan la influencia de los contextos en los que tanto pymes como trasnacionales se ven expuestas en su afán de internalización, para las primeras, e incursión en los mercados locales, para el caso de las segundas. Es más, si se considera que la globalización es un contexto en el que lo global y lo local se vinculan a través de las organizaciones trasnacionales en alianza con las pymes, entonces se abre la posibilidad de discutir las implicaciones que tal contexto tiene en la formación del capital humano y sus competencias laborales.

Las relaciones entre cuadros directivos y empleados se gestan en el marco de las culturas organizacionales con énfasis en la formación de competencias laborales y sus implicaciones para la responsabilidad social y ambiental. En ese sentido, las culturas organizacionales parecen vincularse con las competencias específicas de cuadros directivos y empleados. Así, se advierte una diferencia sustancial entre lo que las organizaciones desean, saben y son capaces de hacer (García, Carreón, Hernández, Valdés, Bustos \& Sandoval, 2017).

Las organizaciones, en tanto entramados de conocimientos, habilidades y valores, permiten la construcción de una responsabilidad social y ambiental, pero al mismo tiempo están determinadas por climas de relaciones y tareas que subsumen sus tradiciones. Ello impediría a las empresas socialmente responsables transitar hacia una cultura sustentable, indicada por competencias en perfecto balance; sin embargo, lejos están las pymes de insertarse en el concierto global y las trasnacionales de participar en el mercado local, ya que sus productos carecen de valor en el sentido cultural más que económico (Carreón, 2016).

Así, a medida que las organizaciones han privilegiado la formación de competencias centradas en el adiestramiento y la capacitación de procesos, están expuestas a elaborar productos y ofrecer servicios que las desvinculan de sus clientes. Tal fenómeno no solo implica deslealtad o desconfianza, sino la pérdida de responsabilidad ante el contexto que las provee de recursos y capitales (Sánchez et al., 2017b). Por ello, en el futuro, la formación del capital humano deberá circunscribirse a la adquisición de valores, normas y creencias que vinculen a las organizaciones con los consumidores. De lo contrario, las empresas tecnificadas y reproductoras de modelos, conocimientos y procesos aspirarán a vender solo a un sector pudiente, que les exigirá aún más innovaciones y valores agregados sin garantizarles su lealtad (Molina et al., 2017). 
La globalización económica supone una serie de lineamientos organizacionales que impactan a los sistemas educativos, principalmente a aquellos en los que la implementación de competencias indica un desarrollo laboral. Antes bien, las organizaciones, al contener al capital humano, construyen un arquetipo de sí mismas conocido como "cultura laboral».

A partir de la cultura organizacional es posible identificar cinco dimensiones relacionadas con la formación profesional y el desarrollo de competencias organizacionales: cooperacracia, adhocracia, competicracia, pretocracia y mercadocracia. La primera alude a la colaboración indicada por su grado de compromiso. La segunda sugiere una dinámica hedonista en la que sus líderes son creativos. La tercera se circunscribe a las competencias en el sentido de producción de conocimiento para la eficiencia de procesos que, por su grado de especialización, requieren de líderes formados en un conocimiento técnico. La cuarta dimensión retoma el orden y su liderazgo conservador como sello distintivo. Por último, la quinta dimensión está ajustada a la dinámica del mercado, ya que sus líderes buscan a toda costa cumplir con los objetivos establecidos (García, Carreón, Hernández, Aguilar \& Rosas, 2016).

La cultura laboral enmarca una serie de principios que guían las relaciones entre los individuos en referencia a sistemas económicos, políticos o sociales. A medida que las organizaciones se circunscriben a procesos de globalidad o localidad, emergen estilos de liderazgo que ajustan los objetivos de las organizaciones a los parámetros de competitividad que el contexto les demanda. A pesar de que los individuos y los grupos a los que pertenecen han adoptado y perfeccionado actitudes, decisiones y acciones, los propósitos organizacionales, al estar vinculados con las estructuras sociales, los sistemas económicos o los regímenes de gobierno, influyen en la construcción de empresas y sus formas de organización (Carreón \& García, 2017).

Las implicaciones que la producción y gestión del conocimiento organizacional tienen para la responsabilidad social y sustentable son diversas si se considera que la cultura y el clima laboral son ejes transversales en los que las competencias se gestan y ajustan a las pymes al mercado global, mientras que insertan a las trasnacionales al ámbito local. A medida que las organizaciones transitan de un querer hacer a una capacidad de hacer, limitan la planificación y flexibilizan sus procesos a fin de que en la formación profesional sus talentos se aproximen al emprendimiento más que a la reproducción de conocimiento (García, 2018).

En este escenario, las culturas organizacionales transforman sus conocimientos en habilidades, pues la entrada de tecnología implica una reproducción de procesos que la capacitación y el adiestramiento pueden solventar. Sin embargo, las competencias laborales, al tener su origen en climas organizacionales poco estructurados, propician que las diferencias salariales entre directivos y empleados se acentúen en términos culturales. En efecto, las organizaciones orientadas al logro parecen soslayar la iniciativa y el compromiso individual o grupal para enaltecer las metas como las principales áreas de oportunidad, que implicarían, paradójicamente, capacidades relativas a la producción de conocimiento, la innovación tecnológica o los procesos competitivos (Carreón et al., 2017).

Es así como las culturas organizacionales se aproximan a la responsabilidad social y ambiental: no como resultado de una gestión de conocimiento o del procesamiento de saberes, sino a partir de vicisitudes derivadas de las diferencias entre ámbitos globales y 
locales, trasnacionales y pymes, cuadros directivos y empleados; o, a nivel personal, las diferencias entre valores, habilidades y conocimientos.

Precisamente, las organizaciones han sabido navegar en la tempestad que implican los mercados y los retos que imponen sus competidores, incluso han establecido alianzas que las obligan a dividir y muchas veces compartir sus ganancias más que sus pérdidas. También han ajustado sus recursos y capitales para satisfacer a sus clientes y socios, y en ese proceso han entendido que la responsabilidad social y ambiental se limita a la producción antes que a la gestión, de forma que es en la construcción de sus culturas donde sus valores han perdido relevancia frente a las habilidades. En tal esquema, donde las competencias se han transmutado en prácticas y procesos que suponen la reproducción de conocimiento más que la innovación y la autonomía, las organizaciones se dirigen hacia la conformidad y la dependencia. De esa manera, la reproducción de modelos organizacionales, planificaciones, estrategias y adiestramiento de procesos indica el estado en el que las organizaciones se encuentran y el futuro que les espera mientras no modifiquen su cultura, valores, normas y creencias (Sánchez et al., 2017a).

En un escenario en el que las competencias son el resultado de la promoción de valores, la producción de conocimiento y la formación de habilidades, las culturas organizacionales se encontrarían en perfecto equilibrio con el clima laboral, la inversión en tecnología y la formación de capital humano. En contraste, en el escenario hacia el que se dirigen las organizaciones que reducen sus culturas a simples protocolos de convivencia, las competencias son solo habilidades que conllevan insatisfacción y deslealtad (Molina et al., 2017).

La cultura organizacional como un factor determinante del desarrollo local establece la influencia del liderazgo motivacional centrado en la inteligencia emocional. En ese sentido, los deseos, saberes y capacidades fungen como condicionantes del emprendimiento de proyectos de fomento empresarial.

Asimismo, en el marco del desarrollo comunitario, la cultura organizacional es un factor que inhibe o potencializa la inserción de las empresas locales en el mercado global, así como la inclusión de las trasnacionales en el mercado local.

En el marco del desarrollo local, en donde prevalecen alianzas estratégicas entre micros, pequeñas y medianas empresas con respecto a las trasnacionales, la imposición de tecnologías y procesos es una práctica común; no obstante, cada vez más una creciente adopción de innovaciones supone una negociación y corresponsabilidad interna en las mipymes que se refleja en sus estrategias de negocios con trasnacionales. De este modo, el presente trabajo ha especificado un modelo a partir de los marcos teóricos, conceptuales y empíricos de la literatura revisada a fin de poder estudiar la innovación organizacional desde hipótesis que, al ser contrastadas, indicarían el grado de innovación organizacional y las áreas de oportunidad subsecuentes (García, Hernández \& Hernández, 2017). Sin embargo, la especificidad de cada organización obliga a la inclusión de otros factores, tales como competitividad o calidad, que la literatura reporta como variables espurias que pueden no tener efecto alguno sobre las variables incluidas en el modelo, pero que en la situación particular de las mipymes determinaría una adopción, implementación o evaluación (Carreón, 2016).

Por otro lado, en el contexto de las políticas educativas de evaluación, certificación 
y acreditación, la influencia del poder central del Estado a través de sus instituciones sobre las organizaciones, sus fases de creación del conocimiento y sus alianzas estratégicas suponen una formación académica, profesional y laboral que convierte al capital humano en activos intangibles.

Carreón y García (2017) especificaron un modelo de acuerdo a la identidad como indicador de la gestión del conocimiento, sosteniendo que la afinidad con el líder y el proyecto son síntomas de un establecimiento de problemas y temas en la agenda de la organización, y advirtiendo que el liderazgo de corte motivacional es el factor reflejante de tal proceso.

Pérez, Valdés y García (2017), por su parte, consideran que la gestión del conocimiento es una continuidad del habitus o las disposiciones organizacionales en contra o a favor de una alternativa innovadora. Es decir, la identidad solo sería el resultado de la transferencia de estos habitus o disposiciones entre líderes y talentos, pero el presente estudio destaca que la motivación —independientemente de su herencia o aprendizajees la ruta que explicaría el proceso racional, deliberado, planificado y sistemático de producción y transferencia del conocimiento en una red entre la universidad pública y la sucursal multinacional.

Son cuatro los factores relativos al crecimiento de las mipymes: capacidad de producción, conocimiento del mercado, diferenciación del producto y logística. Sin embargo, las mipymes tendrían su principal factor multiplicador en la difusión de sus productos y servicios en protocolos digitales como Facebook. En otro estudio, el $60 \%$ de las mipymes no han establecido un organigrama de dirección, producción y control de calidad, y es la percepción del cliente la que determina la compra de productos y la requisición de servicios de las mipymes.

Se puede sostener, entonces, que el conocimiento del mercado determina el crecimiento de las mipymes. En el presente trabajo se observó que los informantes tienen un emprendimiento, innovación y colaboración relacionado con la historia caficultora de la localidad. Asimismo, es innegable que Internet es el difusor por excelencia de las mipymes en su proceso de crecimiento; o que el organigrama es fundamental para delimitar los objetivos, tareas y metas de las mipymes, las cuales, al establecer alianzas, redes y climas, se retroalimentan de la experiencia organizacional de los pares con las que están asociadas. También se puede aseverar que la percepción del cliente es el factor determinante del crecimiento de las mipymes. O que es la historia emprendedora de la localidad y la creatividad de los informantes la que incidió para que la mipymes no solo se mantuviera, sino que además siguiera una trayectoria ascendente (Molina et al., 2017).

\subsection{Instrumentos de la creación del conocimiento}

Los instrumentos que se han empleado para medir el proceso de gestión, producción y transferencia del conocimiento han centrado su atención en las dimensiones de motivación, innovación y comunicación.

La Escala de Inteligencias y Sabidurías (EIS-12) ha demostrado una mayor consistencia interna que validez factorial en virtud de que mide las tres dimensiones, pero al relacionar estos factores con el uso intensivo de tecnologías, dispositivos y redes 
electrónicas, su poder explicativo y predictivo ha sido espurio. En contraste, la Escala de Aprendizaje Organizacional (EAO-12) ha medido más específicamente el proceso de gestión, producción y transferencia del conocimiento, aunque su fiabilidad es menor a la de la EIS-12, pero sus dimensiones han explicado y anticipado un mayor desempeño internauta. No obstante, es la Escala de Gestión del Conocimiento (EGC-21) la que, en su versión corta de 12 ítems, ha establecido una mayor consistencia interna, validez factorial y explicación como predicción del desempeño internauta en organizaciones e instituciones de formación académica, profesional y laboral.

Sin embargo, la composición factorial de las escalas que miden la creación del conocimiento ha sido cuestionada en cuanto a las diferencias que establecen entre organizaciones e instituciones al momento de explicar la relación entre la formación académica, profesional y laboral, principalmente en cuanto a la motivación, la innovación y la comunicación de redes colaborativas. Por consiguiente, es menester abonar a la fiabilidad y la validez con la finalidad de perfeccionar el instrumento y explicar, así como anticipar, escenarios de creación del conocimiento en su proceso de gestión, producción y transferencia.

\section{Método}

En un primer estudio se realizó una investigación no experimental, transversal y exploratoria. Se encuestó a 457 estudiantes de una universidad pública del Estado de México, considerando el semestre en el que realizan prácticas profesionales o bien llevan a cabo su servicio social. El $64 \%$ fueron mujeres y el $35 \%$ hombres. El 58\% dijo tener entre 18 y 22 años, el $24 \%$ entre 23 y 29 años, y el restante $18 \%$ declaró tener más de 29 años. El 34\% dijo que los ingresos de su familia ascendían a menos de 3500 pesos al mes $(M=3241$, $D E=12.35)$, el $51 \%$ declaró un ingreso familiar de entre 3500 y 7000 pesos al mes $(M=$ $5672, D E=124.35)$, y el restante $15 \%$ advirtió que su familia ganaba más de 7000 pesos al mes $(M=8712, D E=235.25)$. Asimismo, el $67 \%$ declaró ser soltero, el $13 \%$ que vivía en unión libre y el $20 \%$ en matrimonio.

En virtud de que la Escala de Gestión del Conocimiento (EGC-12) de Carreón (2016) ha demostrado mayor consistencia interna y validez factorial, y considerando que se edificó en base a la Escala de Sabidurías e Inteligencias (ESI-12) y la Escala de Aprendizaje Organizacional (EAO-12), ambas de García (2019b), principales referentes de fiabilidad y validez, se utilizó una versión corta de 12 ítems que ha sido estandarizada en organizaciones dedicadas a la producción del conocimiento y en alianzas estratégicas con instituciones de educación superior y centros de investigación, ciencia y tecnología.

La Escala de Gestión del Conocimiento de Carreón incluye 21 reactivos alusivos a la motivación, la innovación y la comunicación de acuerdos entre líderes y talentos, aunque se empleó su versión corta debido a su consistencia interna y validez factorial. Cada reactivo incluye las opciones de respuesta: 0 = en nada se parece a mi situación, 1 = se parece muy poco a mi situación, 2 = se parece poco a mi situación, 3 = se parece moderadamente a mi situación, 4 = se parece mucho a mi situación, y 5 = se parece bastante a mi situación. Se utilizó la técnica Delphi para la adecuación cultural del instrumento a la muestra, 
preguntando a un grupo de expertos acerca del significado local de las palabras incluidas en los reactivos e integrando la información en los ítems modificados. Además, se encuestó a la muestra en el vestíbulo de la biblioteca de su universidad, previa garantía por escrito de la confidencialidad de sus respuestas y de que los resultados del presente trabajo no afectarían su estatus académico o laboral.

La información fue procesada en el Paquete Estadístico para Ciencias Sociales (IBM-SPSS-AMOS por su acrónimo en inglés, versión 20.0). Para ello, se estimó la consistencia interna del instrumento con el parámetro alfa de Cronbach y se realizó un análisis factorial exploratorio de ejes principales con rotación promax a fin de establecer la validez del constructo de gestión del conocimiento.

Asimismo, con el objetivo de confirmar la convergencia de los tres factores —motivación, innovación y comunicación del conocimiento—, se procedió a realizar un segundo estudio no experimental, transversal y exploratorio, para lo cual se llevó a cabo una elección no probabilística de 103 estudiantes de una universidad pública. Con ese motivo, se utilizó la misma Escala de Gestión del Conocimiento de Carreón (2016); se prosiguió con la ética y política de resguardo de datos, anonimato y confidencialidad; se utilizó el mismo software para los análisis estadísticos; y se contrastó el modelo con los parámetros de bondad de ajuste (GFI, por su acrónimo en inglés) y residuales (RMSEA, por su acrónimo en inglés).

\section{Resultados}

La tabla 1 muestra las propiedades descriptivas del instrumento. La escala general (alfa de 0.782) obtuvo una consistencia interna superior a la mínima requerida (alfa de 0.700), así como las subescalas de motivación (alfa de 0.781), innovación (alfa de 0.759 ) y comunicación (alfa de 0.774).

Tabla 1. Descriptivos del instrumento

\begin{tabular}{|c|c|c|c|c|c|c|c|c|c|}
\hline Código & & M & D & S & C & A & F1 & F2 & F3 \\
\hline & $\begin{array}{l}\text { Subescala de motivación del } \\
\text { conocimiento }\end{array}$ & & & & & 0.781 & & & \\
\hline MC1 & $\begin{array}{l}\text { Buscar una oportunidad en } \\
\text { multinacionales acorde a mi } \\
\text { estilo de vida }\end{array}$ & 4.01 & 1.02 & 1.03 & 1.82 & 0.767 & & & 0.387 \\
\hline MC2 & $\begin{array}{l}\text { Pedir una oportunidad en mi- } \\
\text { croempresas acorde a mi nivel } \\
\text { de estudios }\end{array}$ & 4.04 & 1.04 & 1.04 & 1.3 & 0.753 & & & 0.346 \\
\hline MC3 & $\begin{array}{l}\text { Exigir una oportunidad en } \\
\text { multinacionales acorde a mi } \\
\text { productividad }\end{array}$ & 4.07 & 1.82 & 1.15 & 1.46 & 0.705 & & & 0.325 \\
\hline MC4 & $\begin{array}{l}\text { Solicitar una oportunidad en } \\
\text { microempresas acorde a mis } \\
\text { ideales } \\
\text { Subescala de innovación del } \\
\text { conocimiento }\end{array}$ & 4.02 & 1.23 & 1.34 & 1.28 & $\begin{array}{l}0.752 \\
0.759\end{array}$ & & & 0.385 \\
\hline
\end{tabular}




\begin{tabular}{|c|c|c|c|c|c|c|c|c|}
\hline IC1 & $\begin{array}{l}\text { Pedir una oportunidad en mul- } \\
\text { tinacionales por la actualización } \\
\text { de mis habilidades }\end{array}$ & 4.07 & 1.25 & 1.03 & 1.04 & 0.732 & & 0.305 \\
\hline IC2 & $\begin{array}{l}\text { Exigir una oportunidad a las } \\
\text { microempresas por la valía de } \\
\text { mis conocimientos }\end{array}$ & 4.15 & 1.81 & 1.25 & 1.26 & 0.721 & & 0.326 \\
\hline IC3 & $\begin{array}{l}\text { Solicitar una oportunidad a } \\
\text { las multinacionales por mi } \\
\text { especialidad }\end{array}$ & 4.35 & 1.01 & 1.25 & 1.36 & 0.703 & & 0.396 \\
\hline IC4 & $\begin{array}{l}\text { Buscar una oportunidad en las } \\
\text { microempresas por la generali- } \\
\text { dad de mis propuestas } \\
\text { Subescala de comunicación } \\
\text { del conocimiento }\end{array}$ & 4.09 & 1.25 & 1.38 & 1.22 & $\begin{array}{l}0.742 \\
0,774\end{array}$ & & 0.382 \\
\hline CC1 & $\begin{array}{l}\text { Solicitar una oportunidad en } \\
\text { multinacionales para difundir } \\
\text { mis propuestas }\end{array}$ & 4.23 & 1.89 & 1.25 & 1.92 & 0.765 & 0.371 & \\
\hline $\mathrm{CC} 2$ & $\begin{array}{l}\text { Pedir una oportunidad en } \\
\text { microempresas para contrastar } \\
\text { mis ideas }\end{array}$ & 4.36 & 1.22 & 1.36 & 1.3 & 0.76 & 0.346 & \\
\hline $\mathrm{CC} 3$ & $\begin{array}{l}\text { Buscar una oportunidad en } \\
\text { multinacionales para perfeccio- } \\
\text { nar mis conocimientos }\end{array}$ & 4.16 & 1.26 & 1.49 & 1.25 & 0.742 & 0.392 & \\
\hline $\mathrm{CC} 4$ & $\begin{array}{l}\text { Exigir una oportunidad en mi- } \\
\text { croempresas para consolidar } \\
\text { mis iniciativas }\end{array}$ & 4.39 & 1.38 & 1.3 & 1.36 & 0.731 & 0.302 & \\
\hline
\end{tabular}

$M=$ media, $\mathrm{D}=$ desviación estándar, $\mathrm{S}=$ sesgo, $\mathrm{C}=$ curtosis, $\mathrm{A}=$ alfa de Crombach. Método de extracción: ejes principales, rotación promax. Adecuación y esfericidad $[X 2=345.34$ (34 gl); $p=0.000 ; \mathrm{KMO}=0.760$ J. F1 = motivación del conocimiento ( $22 \%$ de la varianza total explicada), F2 = innovación del conocimiento (18\% de la varianza total explicada), F3 = comunicación ( $14 \%$ de la varianza total explicada). Todos los ítems se responden con alguna de cinco opciones que van desde 0 = «en nada se parece a mi situación» hasta 5 = "se parece bastante a mi situación».

Fuente: elaboración propia.

Una vez establecidos los tres factores que explicaron el 54\% de la varianza total comentada, se procedió a la estimación de las correlaciones y las covarianzas entre los factores (ver tabla 2).

Tabla 2. Correlaciones y covarianzas entre los factores

\begin{tabular}{l|l|l|l|l|l|l} 
& F1 & F2 & F3 & F1 & F2 & F3 \\
\hline F1 & 1,000 & & & 1 & & \\
\hline F2 & $0.614^{*}$ & 1 & & 0.601 & 1,821 & \\
\hline F3 & $0.482^{* *}$ & $0.336^{*}$ & 1 & 0.572 & 0.712 & 1,792
\end{tabular}

F1 = motivación del conocimiento, F2 = innovación del conocimiento, F3 = comunicación del conocimiento: * $\mathrm{p}<$ $0.01,{ }^{* *} p<0.001,{ }^{* *} p<0.0001$.

Fuente: elaboración propia. 
Es posible observar que los factores mantienen una asociación positiva y significativa, aunque las covarianzas sugieren que están relacionados con otros factores no especificados en el modelo. Adicionalmente, se procedió a estimar el modelo estructural de trayectorias de relaciones reflejantes entre factores e indicadores (ver figura 1).

Figura 1. Modelo estructural de trayectorias de relaciones reflejantes
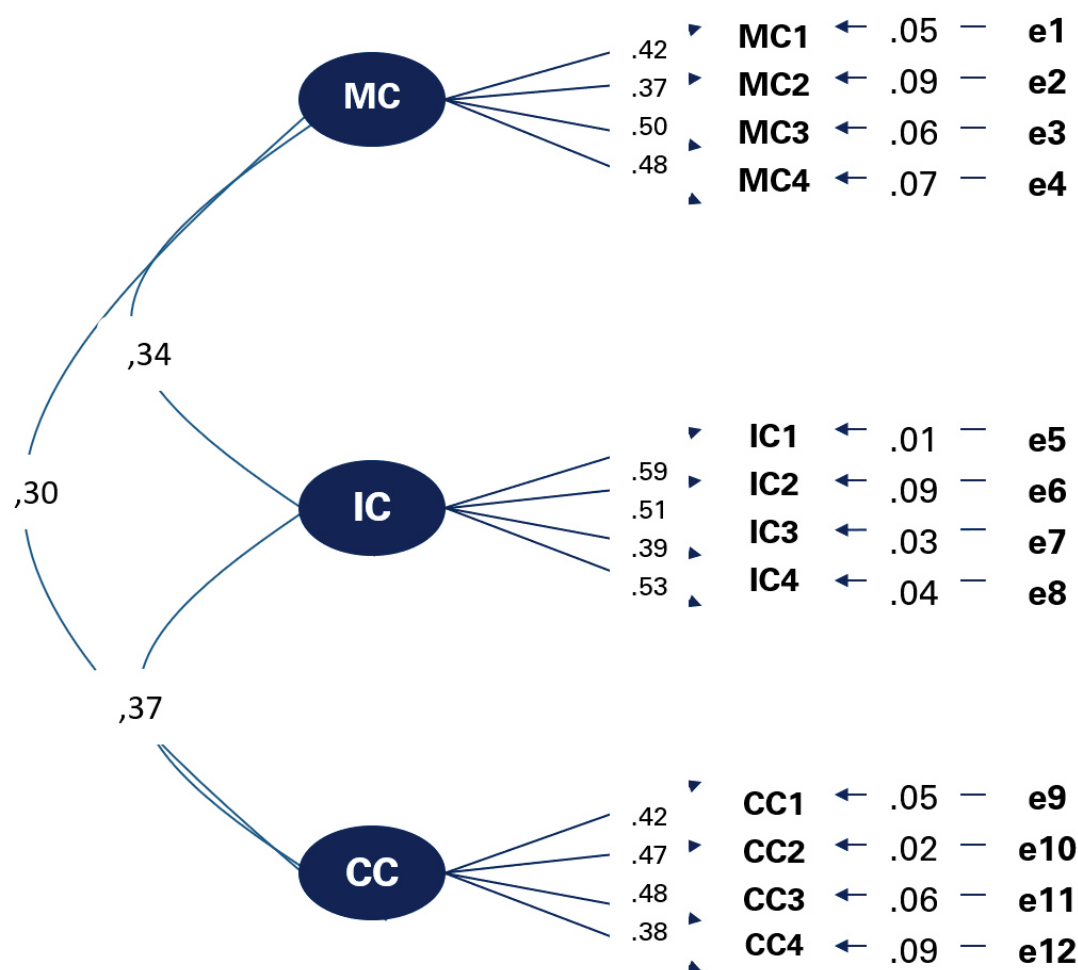

$\mathrm{MC}=$ motivación del conocimiento, $\mathrm{IC}=$ innovación del conocimiento, $\mathrm{CC}=$ comunicación del conocimiento, $\mathrm{e}=$ error de medición del indicador.

Fuente: elaboración propia.

La estructura factorial muestra las asociaciones esperadas entre los factores ya que, se supone, son indicadores de un factor de segundo orden, como es el caso de la creación del conocimiento. Sin embargo, tal solución factorial solo explica el $54 \%$ de la varianza, indicando la inclusión de otros factores relativos a las relaciones colaborativas como la empatía, el compromiso o la satisfacción, que podrían incrementar el porcentaje de la varianza total explicada del constructo, aun cuando los parámetros de ajuste $\lceil X 2=124.35$ $(23 \mathrm{gl}), \mathrm{p}=0.007, \mathrm{GFI}=0.990, \mathrm{CFI}=0.995$ y RMSEA $=0.009 \mathrm{~J}$ sugieren la aceptación de la hipótesis nula relativa a la explicación de la creación del conocimiento desde las teorías de la gestión, la producción y la transferencia. 


\section{Discusión}

El aporte del trabajo al estado del conocimiento radica en la discusión de los factores que permitan un mayor entendimiento de la conformidad y la obediencia como paradigmas limitadores del emprendimiento. Otro aporte del presente trabajo radica en la interpretación de los significados de informantes emprendedores con respecto a las categorías de alianzas estratégicas, redes de conocimiento y clima de innovación, así como en la especificación y contrastación de un modelo de gestión de conocimiento, cuya confiabilidad y validez debió establecerse con la finalidad de demostrar la hipótesis nula relativa al ajuste de las relaciones teóricas con respecto a las observaciones ponderadas.

Los límites del presente estudio son: 1) el tipo de investigación exploratoria, transversal y cualitativa al encontrar que la historia emprendedora de la localidad y la creatividad inciden en el emprendimiento, la innovación y la colaboración de las mipymes; 2) la interpretación de los significados de alianza, conocimiento e innovación al asumir que estos son los procesos que orientan el cambio de las mipymes, soslayando sus usos y costumbres; 3 ) las diferencias con el estado del conocimiento en cuanto a la gestión y la administración de las tres categorías en comento; y 4) el tipo de selección muestral no probabilística e intencional, así como el análisis factorial exploratorio, limitan los resultados obtenidos a la muestra de estudio.

En relación con la literatura consultada, esta ha demostrado que la creación del conocimiento se gesta en: 1) organizaciones orientadas al equilibrio entre las demandas externas y los recursos internos; 2) organizaciones con culturas flexibles, liderazgos transformadores, comunicación bidireccional y motivación intrínseca; 3) organizaciones con estándares de calidad de sus procesos consistentes en la evaluación, acreditación y certificación; y 4) organizaciones con estructuras postburocráticas centradas en la gestión más que en la administración de las oportunidades y las capacidades de sus líderes y talentos. Adicionalmente, el presente trabajo ha demostrado que: 5) las organizaciones creadoras de conocimiento derivan de tres factores; a saber, motivación, innovación y comunicación.

Por consiguiente, la motivación, innovación y comunicación del conocimiento están vinculadas más a las alianzas estratégicas entre organizaciones e instituciones formadoras del capital intelectual en su perspectiva de activo intangible, ya que, si los estudiantes tienen un excelente desempeño académico, es posible que reproduzcan sus competencias en el ámbito laboral; no obstante, la relación bidireccional con sus grupos colaborativos sugiere una motivación constante, una innovación permanente y una comunicación eficaz.

En el caso de la innovación de procesos frente a la optimización de recursos como ventaja competitiva de las pymes locales, la formación académica, profesional y laboral, al estar orientada hacia la motivación y la comunicación, sugiere códigos para la transferencia del conocimiento del capital intelectual especializados en los futuros talentos.

Se recomienda: 1) llevar a cabo un estudio cuasi experimental para establecer la incidencia de factores que por su control podrían develar el grado de emprendimiento, innovación y colaboración; 2) establecer trayectorias de relaciones de dependencia entre las variables en comento; 3) implementar el efecto de otras variables, como el 
microfinanciamiento, para observar los cambios en un periodo de tiempo determinado; y 4) el contraste del modelo con otros contextos y muestras diferentes a la de estudio.

\section{Conclusiones}

El objetivo del presente trabajo fue especificar un modelo para el estudio de la generación del conocimiento en una universidad pública del centro de México, con formación y prácticas laborales en sucursales de multinacionales líderes del mercado local.

A partir de una revisión de la literatura indexada a repositorios líderes de América Latina como Dialnet, Latindex y Redalyc; la interpretación de los discursos de informantes; y el establecimiento de la confiabilidad y validez de un instrumento que midió tres factores indicativos de la generación del conocimiento, se especificó un modelo. Sin embargo, el porcentaje de la varianza total explicada sugiere la inclusión de otros factores, relativos a la empatía, el compromiso y la satisfacción, que podrían incrementar el porcentaje de la varianza total explicada, aunque la revisión de la literatura y el análisis de los discursos de informantes claves no los aborden.

Además, es menester llevar a cabo el contraste del modelo en instituciones de educación superior con un sistema de formación y prácticas laborales a fin de poder evidenciar los alcances y límites del modelo especificado con o sin la inclusión de los factores relativos a la empatía, el compromiso y la satisfacción. 


\section{bibliografía}

Abdiaziz, M., \& Yassin, A.

2014

Corporate Innovation and Organizational

Performance: The Case of Somalia

Telecommunication Industry. Proceding

Kuala Lumpur International Business,

Economics and Law Conference, 4(1),

260-271.

\section{Carreón, J.}

Desarrollo humano: gobernanza y

emprendimiento social. México:

Universidad Nacional Autónoma de

México, Escuela Nacional de Trabajo Social.

\section{Carreón, J., \& García, C.}

2017 Specification of a Model for Study of Vocational Training and Job. International Journal of Advances in Social Sciences and Humanities, 5(6), 13-18.

\section{Carreón, J., Hernández, J.,} García, C., \& Bustos, J. M.

2017 Factores perceptuales del neoliberalismo educativo en una IES del centro de México. Perspectiva, 15, 50-57.

\section{Carreón, J., Hernández, J.,} Quintero, M. L., García, C., \& Mejía, S.

2016 Redes de conocimiento en torno a la complejidad organizacional: aprendizaje de la autoregulación, disipación, adaptabilidad y dinamismo. Prospectivas en Psicología, 2(2), 57-70

Cherubini, A., Barbieux, D., Reichert, F. M., Tello, G., \& Zawislak, P. A.

Innovation and Dynamics Capabilities of the Firm: Defining and Assessment Model. Administración de Empresas, 57(3), 232-

\section{Cleger, S.}

2016 TIC inteligentes en la innovación educativa. Científica, 26, 143-148.

\section{Conto, F., Fiore, M., \& La Sala, P.}

2013

The Role of Innovation in the Integration

Processes of Integrated Projects of Food

Chain: The Case of the Cherry Cultivation

Chain in Apulia Region. Intellectual

Economics, 7(18), 467-485.

Crossan, M., \& Apaydin, M.

2010 A Multi-Dimensional Framework of Organizational Innovation: A Systematic Review of Literature. Journal of Management Studies, 47(6), 11541191. doi: http://doi.org/10.1111/j.14676486.2009.00880.x

De Mello, M., Marx, A., \&

\section{Salerno, $\mathbf{M}$.}

2012 Organizational Structures to Support Innovation: How Do Companies Decide? Administración e Innovación, 9(4), 5-21. Recuperado de: https://www.redalyc.org/ pdf/973/97324897002.pdf

\section{García, C.}

Modelo de millennials emprendedores. Cambios y Permanencias, 8(2), 379-395.

\section{García, C.}

2018a Emprendimiento caficultor en migrantes de la región Huasteca del centro de México.

Equidad y Desarrollo, 30, 119-147.

\section{García, C.}

2018b Interpretaciones de los discursos de gestión del conocimiento para la comprensión de narrativas de emprendimiento innovador. Inclusiones, 5, 96-111. 


\section{bibliografía}

García, C.

$2019 a$

Configuración factorial exploratoria

de la percepción de oportunidades

emprendedoras. Caleidoscopio, 41, 1-13

García, C.

$2019 b$

Inteligencias y sabidurías organizacionales:

redes de conocimiento en torno al

aprendizaje de la complejidad. Psicogente,

22(41), 1-28.

García, C., Carreón, J.,

Hernández, J., Mendoza, D., Mejía, S., \& Quintana, L. D.

Emprendimiento digital: estudio de caso

con universitarios de comunicación en

la Universidad Autónoma del Estado de

México. Visión Gerencial, 14(2), 187-300.

García, C., Carreón, J.,

Hernández, J., Aguilar, J. A., \&

Rosas, F. J.

2016

Contraste de un modelo de calidad de

vida en estudiantes de la Universidad

Autónoma del Estado de México. Visión

Gerencial, 15(1), 8-43

García, C., Carreón, J., \&

Hernández, J.

2017 Especificación de un modelo de estudio sobre emprendimiento para jefas de familia. Educere, 21(70), 679-685.

García, C., Carreón, J.,

Hernández, J., Bustos, J. M., \& Quintero, M. L.

Expected and perceived governance in a social rehabilitation center from Mexico

City. International Journal of Advances in Social Science and Humanities, 5(5), 6-13.
García, C., Carreón, J.,

Hernández, J., Valdés, O.,

Bustos, J. M., \& Sandoval, F. R.

2017

Agenda of the governance water services

in a demarcation of Mexico City. Global

Advances Research Journal of Agricultural

Science, 6(10), 1-17.

García, C., Hernández, G., \&

Hernández, J.

2017

Un análisis conductual en Millennials

usuarios de telefonía móvil. Encuentros

Multidisciplinares, 19(57), 1-8.

García, C., Quintero, M. L., \&

Carreón, J.

2017

Emprendimiento micro-caficultor en

migrantes de San Luis Potosí, centro de

México. Margen, 87, 1-15.

García, C., Bustos, J. M.,

Carreón, J., \& Hernández, J.

2018

Especificación de un modelo para el

estudio de las expectativas desde

su función mediadora. Enseñanza e

Investigación en Psicología, 23(1), 75-81.

Genesi, M., Romero, N., \&

Tinedo, $Y$.

201

Comportamiento organizacional del talento humano en las instituciones educativas.

Negotium, 18, 102-128.

\section{Gómez, F.}

2010

Competencias profesionales en trabajo social. Portularia, 10, 51-63.

\section{González, R., \& García, F.}

Innovación abierta: un modelo preliminar desde la gestión del conocimiento. Inteligible Capital, 7(1), 82-115. doi: http:// doi.org/10.3926/ic.2011.v7n1.p82-115 


\section{bibliografía}

Guedes, S. M., Loiola, E., Andrade, F., \& Da Costa, S. C.

2015

Creativity and Innovation as Defined by

Workers. Administración, 21(3), 549-576.

Recuperado de: https://www.redalyc.org/

pdf/4011/401143287001.pdf

\section{Henao, R., \& Londoño, A.}

2012

Diseño de un modelo de dirección por competencias distintivas para las pymes exportadoras. Semestre Económico, 15, 197-223.

\section{Jalonen, $\mathbf{H}$.}

The Uncertainty of Innovation: A Systematic Review of the Literature. Journal of Management Research, 4(1), $1-47$.

\section{Lewandoski, $\mathbf{M}$.}

How to monitor the effects of managerial innovation in public cultural institutions. Knowledge Management \& Innovation, 1 , 19-21.

Luoma-aho, V., Lappalainen, R., Uusitalo, O., Vos, M., Lämsä, A-M.,... Maaranen, P.

Added Value of Intangibles for

Organizational Innovation. Human

Technology, 8(1), 7-23. doi: http://doi.

org/10.17011/ht/urn.201205141650

\section{Marques, J.}

2014 Closed versus Open Innovation: Evolution or Combination? International Journal of Business and Management, 9(3), 196-203. doi: http://doi.org/10.5539/ijbm.v9n3p196

Molina, H. D., Cruz, M. G., Carreón, J., \& Rojano, S. M. in the Organization: A Reflection on

a Mexican Photovoltaic Industry.

An Empirical Framework Proposal.

International Journal Advances in Social

Science \& Humanities, 5(11), 1-4.

Pérez, G., Valdés, O., \& García, C.

2017 Determinantes del habitus académico a partir de la gestión del conocimiento. Margen, 85, 1-12.

\section{Prieto, M., \& Villamor, P.}

2012 Libertad de elección, competencia y calidad. Revista de Curriculum y Formación del Profesorado, 16, 150-162.

\section{Rodríguez, J.}

Los rincones de trabajo en el desarrollo de competencias básicas. Revista Docencia e Investigación, 21, 105-130.

Rodríguez, R., \& Hechanova, M. 2013

A Study of Culture Dimensions,

Organizational Ambidexterity, and

Perceived Innovations in Team. Journal of Technology Management and Innovation, 9(3), 21-33.

Rodríguez, R., Moreno, B., De Rivas, S., Álvarez, A., \& SanzVergel, A.

2010 Positive Psychology at Work: Mutual Gains for Individuals and Organizations. Revista de Psicología del Trabajo y las Organizaciones, 26(3), 235-253. doi: http:// doi.org/10.5093/tr2010v26n3a7

\section{Roger, E.}

Theory of Innovation Diffusión. Nueva York: Prentice Hall. 


\section{bibliografía}

Sánchez, A., Cruz, M. G.,

Carreón, J., \& Molina H. D.

2017

Model a Perception of Entrepreneurships:

A Reflection on a Mexican Coffee Industry

Through an Empirical framework proposal.

Social Science Learning Education Journal,

2(11), 1-10.

Sánchez, A., Quintero,

M. L., Sánchez, R., Fierro, E., \&

García, C.

2017

Gobernanza del emprendimiento social:

especificación de un modelo para el

estudio de la innovación local. Nómadas,

$51,1-21$

\section{Secanella, J.}

Nuevas competencias para la

empleabilidad de los estudiantes

universitarios. Cuestión Universitaria, 7,

40-47.

Torrelles, C., Coiduras, J., Isus,

S., Carrera, X., París, G., \&

\section{Cela, J.}

2011 Competencia de trabajo en equipo:

definición y categorización. Revista de

Curriculum y Formación del Profesorado,

15, 329-344,

\section{Urbancová, H.}

2013 Competitive Advantage Achievement

Through Innovation and Knowledge.

Journal of Competitiveness, 5(1), 82-96.

doi: http://doi.org/10.7441/joc.2013.01.06

\section{Vázquez, E., Carreón, J., \&}

\section{Sánchez, A.}

Knowledge Around the Strategies Alliances

of Micro Coffee Producers. International

Journal Environment, Agriculture \&

Biotechnology, 2(6), 3294-3302.
Villegas, E., García, C., \& Hernández, T. J.

Establecimiento de una política de ciencia y tecnología para la incubación

de microempresas innovadoras del conocimiento. Inclusiones, 5, 19-26.

Fecha de recepción: 19 de abril de 2018

Fecha de aceptación: 30 de enero de 2019 